\title{
First results of the Trip-t based T2K front end electronics performance with GM-APD
}

\author{
A. Vacheret*, M. Noy and M. Raymond \\ Imperial College London, SW7 2AZ London, UK \\ E-mail: matthew.noyeimperial.ac.uk, m.raymondeimperial.ac.uk, \\ a.vacheret@imperial.ac.uk
}

\section{A. Weber}

CCLRC - Rutherford Appleton Laboratory, Chilton, Didcot, OX11 OQX, UK

E-mail: A.J.G.Weber@rl.ac.uk

The TRIP-t front end board (TFB) will read out Geiger-mode multi-pixel Avalanche Photo-Diodes (GM-APD) in several T2K detector systems. We report the first tests of the TFB electronics with GM-APD. Measurements of GM-APD charge spectra, energy and time resolution have been performed. Individual channel voltage trimming circuit functionality has also been tested. These results indicate very good performance of the TFB prototype with both 400 and 100 pixels GMAPD from Hamamatsu photonics.

International workshop on new photon-detectors PD07

June 27-29 2007

Kobe University, Kobe, Japan

\footnotetext{
* Speaker.
} 


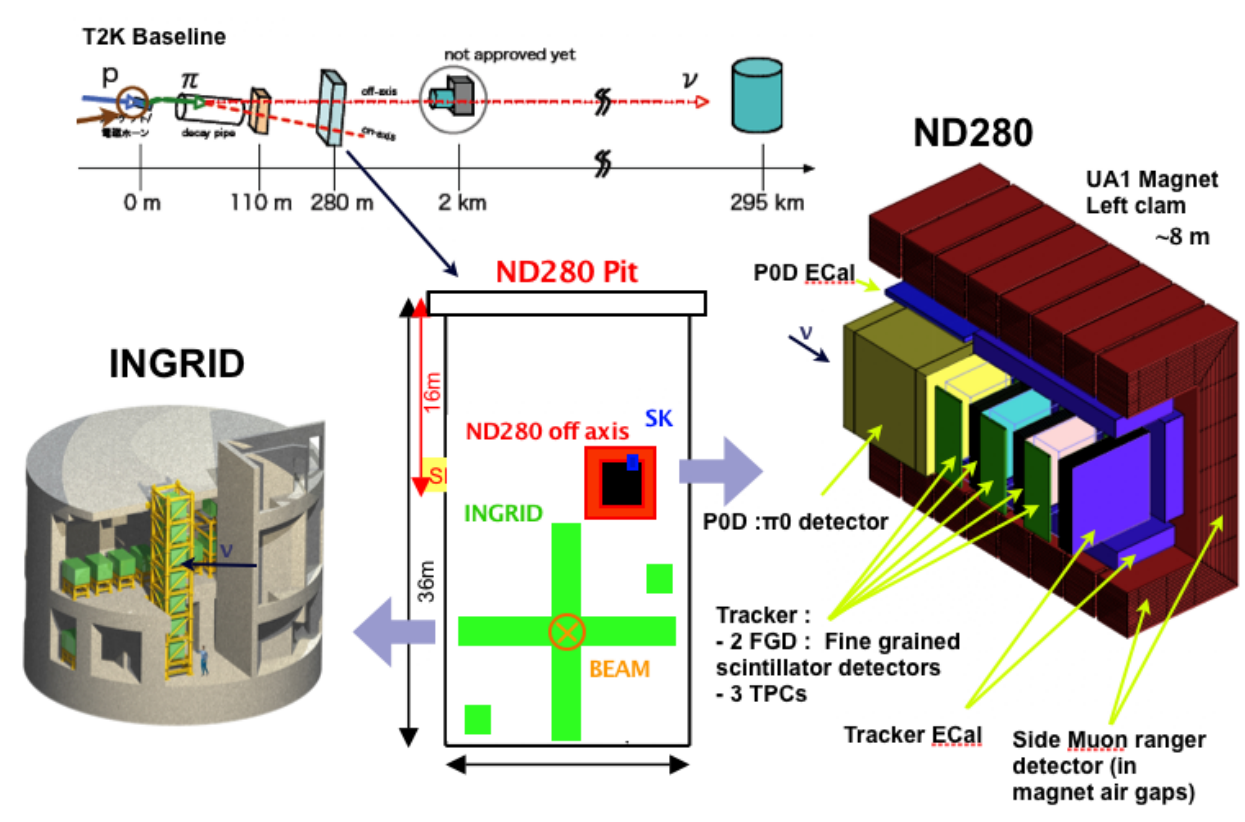

Figure 1: The T2K baseline (top) and a cut view of the ND280 pit with both detectors positions in respect to the beam. On the left is shown a 3D view of the on-axis detector (INGRID). On the right, a cut view of the $280 \mathrm{~m}$ off-axis detector showing the UA1 right clam and the different detector sub-systems.

\section{Introduction}

The Tokai-to-Kamioka (T2K) experiment [1] is a second generation long baseline neutrino oscillation experiment that will take place in Japan. The new $40 \mathrm{GeV}$ proton synchrotron under construction at the J-PARC facility in Tokai will produce a $600 \mathrm{MeV}, 0.75 \mathrm{MW}$ neutrino beam directed towards the far detector, Super-Kamiokande (SK) [2] located $295 \mathrm{~km}$ away in the Kamioka mine. The main goals of $\mathrm{T} 2 \mathrm{~K}$ are the precise measurements of the oscillation parameters $\Delta m^{2}$ and $\theta_{23}$ and the search for a non-zero $\theta_{13}$. The high statistics of neutrino events expected at SK will allow for a measurement of $\Delta m^{2}$ and $\theta_{23}$ at the few percent level by observing the disappearance of $v_{\mu}$ events. The sensitivity on $\sin ^{2} \theta_{13}$ will be improved by a factor of ten from the current limit. T2K will start data taking in April 2009 and will run for five years.

The configuration of the T2K beamline is illustrated at the top of Fig. 1. An off-axis beam technique will be used to produce a narrow peaked and low contamination neutrino beam. The off-axis angle will be set between 2 and $2.5^{\circ}$ to maximize the sensitivity at the far detector. A set of near detectors will measure the off-axis angle, the neutrino energy spectrum and backgrounds events before oscillations. A complex of near detectors at a distance of $280 \mathrm{~m}$ from the target station are currently under construction and a second complex of detectors is proposed at $2 \mathrm{~km}$. A cut view of the $280 \mathrm{~m}$ pit with both near detectors is shown in Fig. 1. On-axis, a grid of iron-scintilator detectors (INGRID) will be used to monitor the on-axis beam profile and flux by measuring charge current neutrino interactions and will measure precisely the off-axis angle. The $280 \mathrm{~m}$ off-axis detector (ND280) system will be situated inside the UA1 magnet and comprises scintillator based sub-systems (P0D, FGD, ECAL, SMRD) and 3 TPC modules. A 0.2 T dipole field will help separating "wrong sign" charge current interactions. Fine grained scintillator detectors will act as 

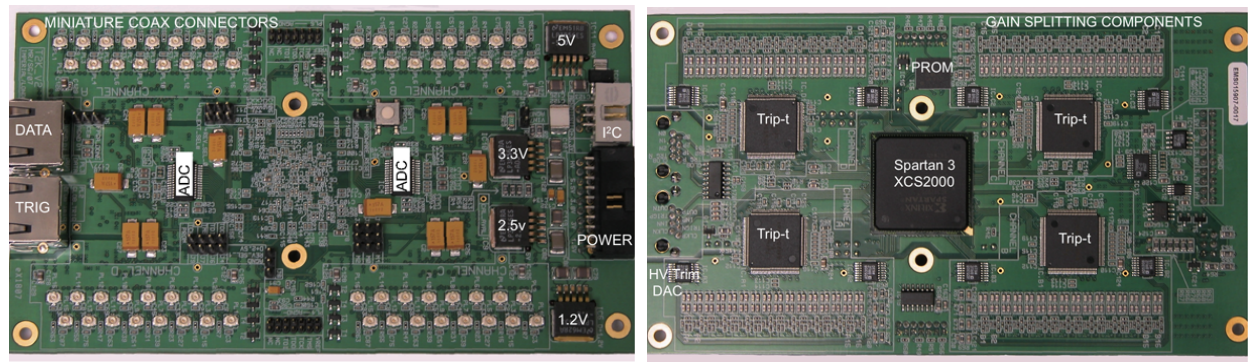

Figure 2: Views of both side of the TFB with details of the components.

high density targets for neutrino interactions whereas the TPC will be used for tracking escaping particles.

The requirements for the readout at the ND280 are the following :

- The photosensor response should be insensitive to magnetic field

- Photon counting capability is required due to low light yield and relatively large dynamic range $\sim 400$ photon equivalent ( $p e$ ) is needed in both target detectors and calorimeter region.

- Readout should be of small dimension (tight space constraints)

- Low maintenance (5 years operation) and low power consumption

The first requirement rules out the use of classic multi-anode PMT. The novel Geiger-Mode Avalanche Photo-Diode (GM-APD) devices, however, are insensitive to magnetic field and match all important ND280 requirements. All ND280 scintillator-based detectors will be instrumented with GMAPD for a total of 70000 channels.

The J-PARC proton beamline will produce a spill every $2.3 \mathrm{~s}$. The current planned spill time structure consists of 9 buckets of $58 \mathrm{~ns}$ separated by $540 \mathrm{~ns}$ gaps. The occupancy of physics signal is expected to be 0.5 interaction/spill/ton of scintillators in the target detectors. Despite an average of a dozen of neutrino interactions per spill expected in the 1kT UA1 magnet iron, the conjunction of finely segmented scintillator detectors and a reasonably low occupancy of signal per channel per bucket keep the design of the readout system relatively simple. In the next section, the front end electronics, designed to readout GM-APD for several near detector systems is described.

\section{The TRIP-t Front end Board}

The front end electronics is based on the Fermilab Trip-t ASIC [4]. Originally designed for the D0 experiment at the Tevatron, this chip contains all the necessary features required to read out GM-APD in the T2K near detectors. The prototype TFB is shown in figure 2 where the main components are indicated. An FPGA sequences the operation, digitization and readout of four Trip- $t$ chips, formatting and transmitting the data off-board.

Figure 3 shows a block diagram of the 32 channel Trip-t chip. The integration period of the preamplifier is controlled by a reset signal. The preamplifier output is taken to an analogue pipeline and 


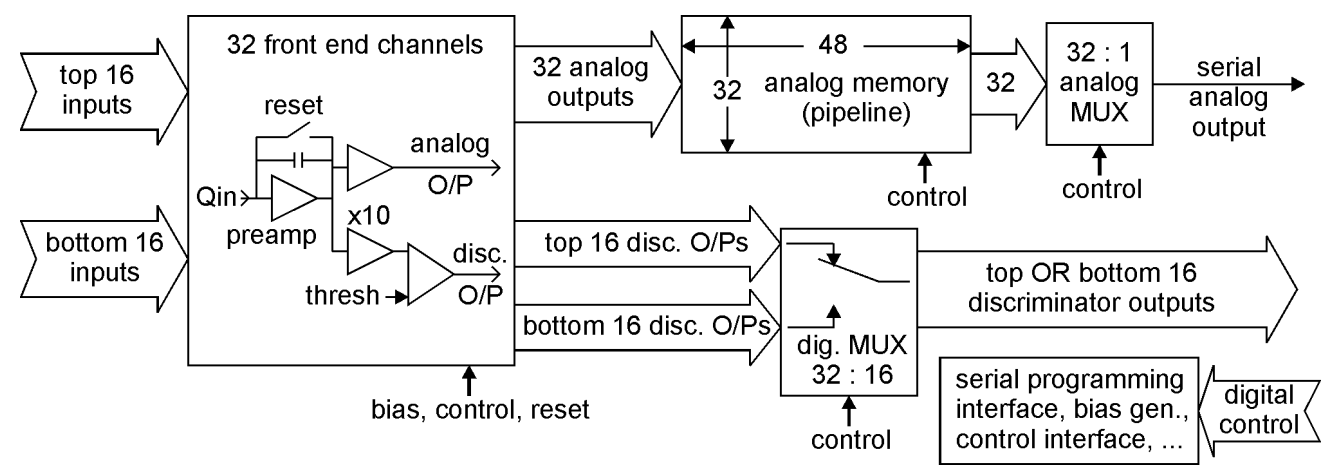

Figure 3: Block diagram of the Trip-t chip.

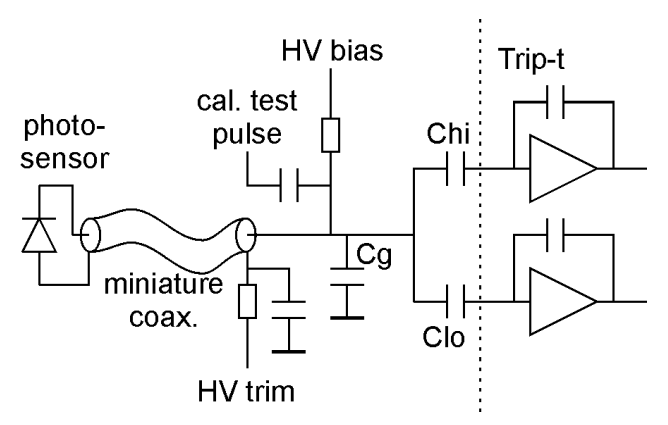

Figure 4: Schematic of circuit to divide GM-APD charge between high and low gain channels.

a discriminator with a programmable threshold voltage. In T2K the preamplifier integration time will be synchronized with the beam structure (reset between bunches, minimum $50 \mathrm{nsec}$ ) and the pipeline used to store the samples. The contents of the pipeline can be read out, digitized and transmitted off-board during the spill gap.

The full range of signals cannot be accommodated while simultaneously allowing sufficient precision in the discriminator setting, so two Trip-t channels are used for each device with the signal capacitively divided (figure 4 ) to provide high and low gain channels. The chip architecture (fig. 3 ) is such that the discriminator outputs can only be selected in groups of 16 for transmission offchip, but this presents no problem since we are only interested in the discriminator outputs of the 16 high gain channels, so these are permanently selected for transmission to the FPGA where time stamping is implemented. The discriminators are reset during the preamplifier reset period. Any timestamps generated are included in the TFB output data.

The TFB also provides the slow control functionalities of bias voltage trimming to individual GMAPD devices, electronic channel calibration, and temperature and voltage monitoring.

\section{Measured performance of the TFB with GM-APD}

Measurements were performed on Hammamatsu Multi-Pixel Photon Counter (MPPC) [3] 100 and 400 pixels connected to the TFB with miniature coaxial cable. A schematic of the setup is illustrated in Fig. 5. The TFB was read out using a customized interface card and a PC with 


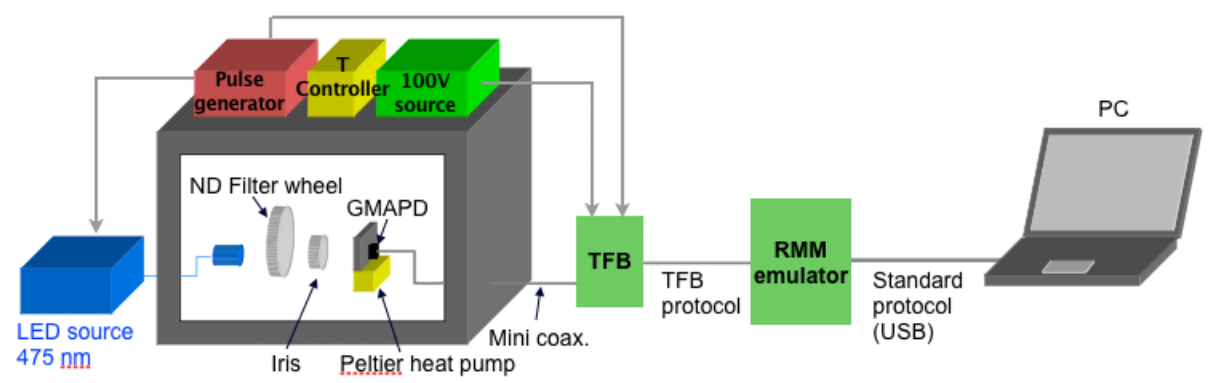

Figure 5: Schematic of the test bench used for MPPC and electronics readout tests. The setup comprises a fast LED source, a dark box with filters, optical components and a temperature controlled stand for the MPPC. The LED source is triggered externally by the clock signal from the TFB. The MPPC signal is readout with the TFB and the data is acquired via an interface card connected to PC using standard USB protocol.

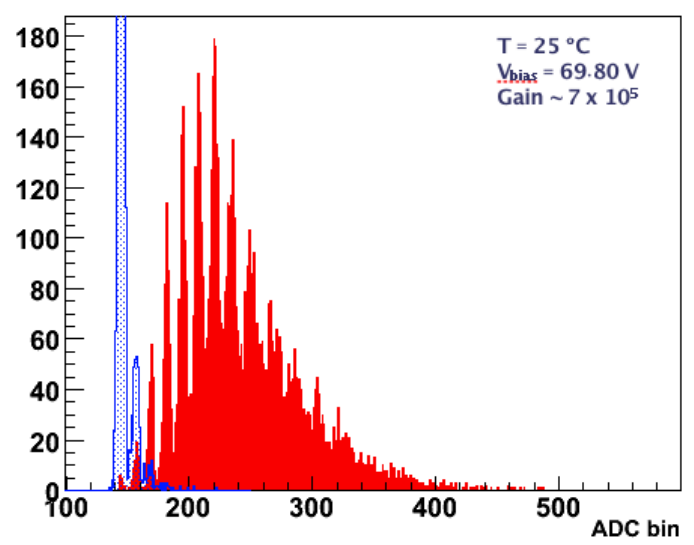

Figure 6: (a) ADC spectra of the high gain channel. The first peak is the pedestal.

the Labview program. The MPPC temperature was controlled with a Peltier heat pump to $0.1^{\circ} \mathrm{C}$ accuracy. A stabilized $475 \mathrm{~nm}$, nanosecond pulse LED source in combination with neutral density filters and an iris were used to select different level of light intensity. In the following sections, we present measurements of charge spectra, time resolution and MPPC parameters versus voltage.

\subsection{MPPC charge spectra}

The signal charge resolution and dynamic range have been checked with both types of MPPC using the LED pulse. The TFB was set to 10 integration window of $250 \mathrm{~ns}$ with a reset period of 100ns. The LED flash was set to occur in the second window, $150 \mathrm{~ns}$ after the start of the gate. Fig. 6 shows typical high gain channel ADC spectra for LED off and low LED intensity corresponding to 5 pe. The gain of the channel has been tuned to give 0.1 pe resolution at $\mathrm{G}=5 \times 10^{5}$ and a clean separation of peaks up to 12 pe can be seen from the distribution. Similar peaks resolution was achieved with 100 pixel MPPC at a gain of $10^{6}$. Fig. 7 shows ADC spectra for large LED 

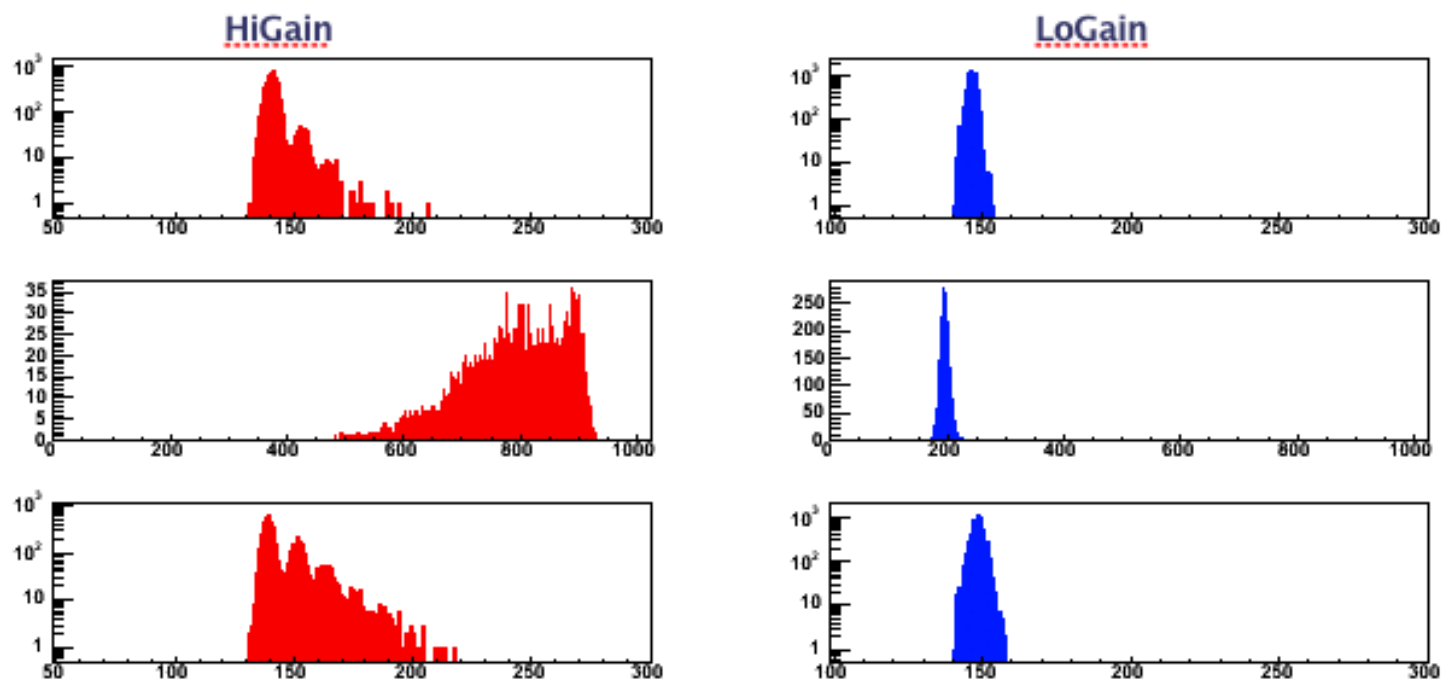

Figure 7: First 3 time window ADC spectra for high and low gain channel. The MPPC is illuminated with a high intensity LED pulse in the $2^{\text {nd }}$ window (middle plots). Both first and last time window is on logarithmic scale. MPPC was biased at $69.80 \mathrm{~V}$ recommended voltage and kept at $25^{\circ} \mathrm{C}$.

intensity pulsing of the order of $40 \mathrm{pe}$. Signal from MPPC nanosecond scale after-pulses integrated in the following time window is observed in the bottom left ADC spectrum. The phenomenon of after-pulses in MPPC is studied extensively in other presentations of this conference. Figure 7 illustrates the complementarity of both channels to ensure good energy resolution for calibration and dynamic range for physics signals.

\subsection{Time resolution}

The TFB is sequenced with a $100 \mathrm{MHz}$ clock. Using 4 phases shifted by $90^{\circ}$ allows the TRIP-t discriminator output to be time-stamped with 2.5 ns resolution in the FPGA. The TRIP$\mathrm{t}$ discriminator firing time depends on the total input charge signal and the speed with which it arrives, giving rise to a time walk effect. The time constant of the MPPC pulse is of the order of $10 \mathrm{~ns}$ for 400 pixels devices and around 30-50 ns for 100 pixels devices. Timestamp distributions versus integrated charge have been studied and preliminary estimations of time resolution have been extracted.

A large interval of charge value has been spanned by pulsing the LED source with different neutral density filter settings. In figure 8 , the timestamp values dependance on signal size for the high gain channel is shown for all LED intensity values. The discriminator threshold was set to 2.5 pe. The dependance of the discriminator time walk with charge can be seen for ADC values close or equivalent to $2.5 \mathrm{pe}$. The time resolution can be estimated for signal above $5 \mathrm{pe}$ as a Gaussian dispersion in a $2.5 \mathrm{~ns}$ discrete window : $T_{\text {res }}=2.5 / \sqrt{12} \approx 1$ ns. In the ND280 detectors, the scintillator bars are read out using Wavelength Shifting (WLS) Fibre (coupled to MPPC) and the time jitter introduced by these fibres is of the order of $10 \mathrm{~ns}$. Since the time resolution of the 


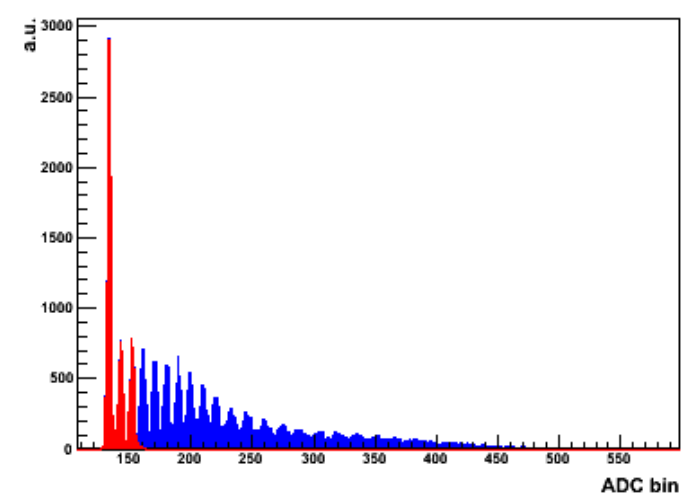

(a)

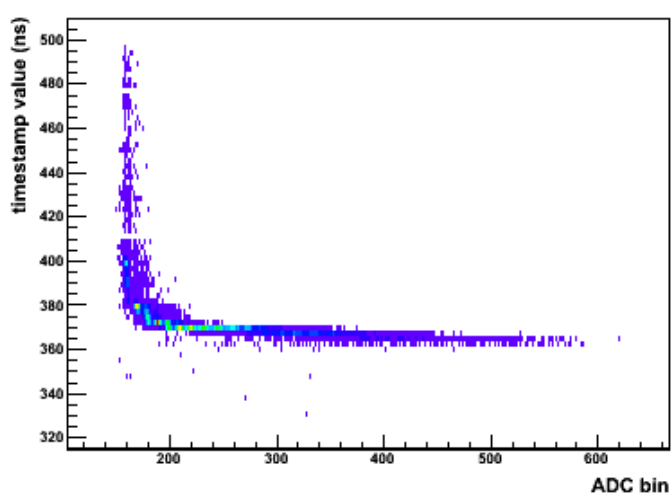

(b)

Figure 8: (a) Sum of charge spectra for all LED intensity (blue). The region in red corresponds to the ADC values below the discriminator threshold and confirm the value of 2.5 pe set for that measurement. (b) shows the correlation between timestamp values and integrated charge.

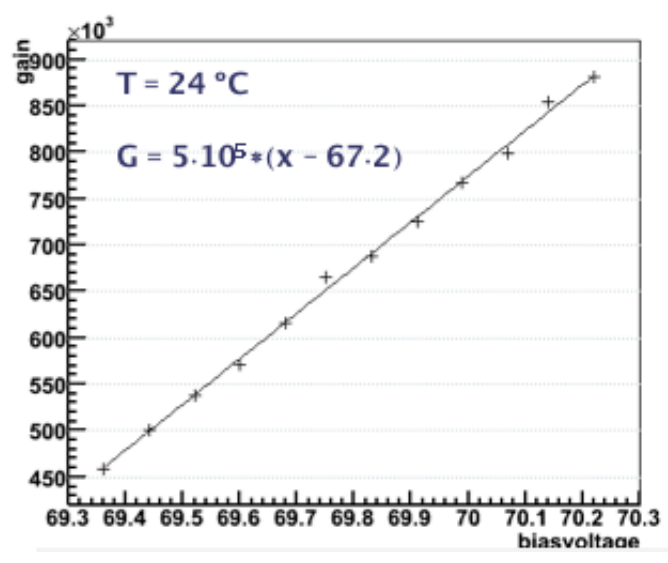

(a)

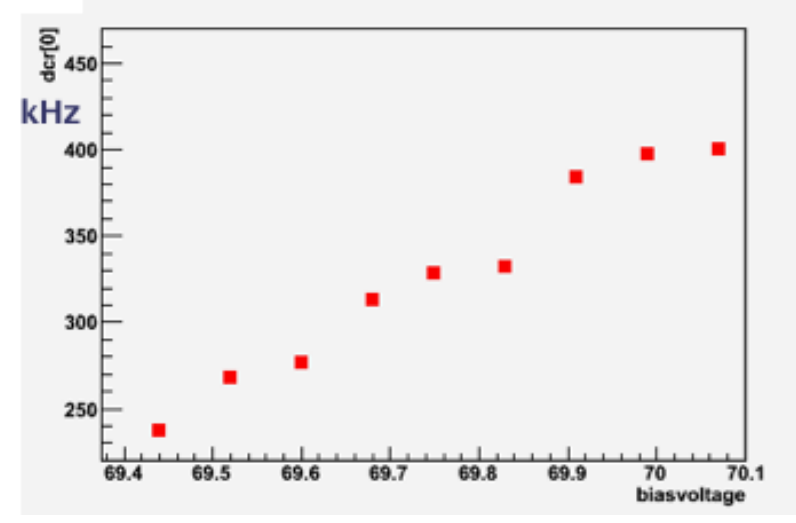

(b)

Figure 9: (a) Gain values calculated using distance inter-peak. (b) Dark count rate estimation using fraction of events above 0.5 pe in ADC spectrum. For this measurement, HV increments of $80 \mathrm{mV}$ have been used but this could be reduced to $20 \mathrm{mV}$ if necessary.

MPPC/TFB system alone is much better that this, it is clear that the WLS fibre time fluctuations will dominate the overall system time resolution.

\subsection{Using the HV trimming functionality}

An HV trim circuit will be used to adjust the individual MPPC bias voltage on the board to achieve the same gain. This can also be used to perform voltage scans to test and calibrate MPPC. The 8 bit DAC allows for values over a $5 \mathrm{~V}$ range with discrete steps of $20 \mathrm{mV}$. The temperature dependance of MPPC being around $50 \mathrm{mV} /{ }^{\circ} \mathrm{C}$, variations of temperature could be corrected as well 
as voltage drifts. The spread in over-voltage for MPPC is less than $1 \mathrm{~V}$ and the current range of voltage should accommodate comfortably device to device variations as well as large temperature gradients in the detectors. Gain and dark count rate have been measured as a function of bias voltage and results are shown in figure Fig. 9. Both values of gain and dark count rate are consistent with manufacturer values. The MPPC breakdown voltage has been extracted from a linear fit of the gain curve and found to be equal to $V_{b d}=67.20 \mathrm{~V}$ at $24^{\circ} \mathrm{C}$.

\section{Summary}

The TFB will be used in the T2K near detectors to readout MPPC. We report the first measurements of energy and time response of the prototype TFB with MPPC. The performance of the board has proved to be very good. Charge resolution, noise, dynamic range and time resolution meet the T2K near detectors requirements. The HV trim circuit functionality has also been verified.

\section{Acknowledgments}

The presenting author would like to express gratitude to the organizers of PD07 for their invitation to the conference and their hospitality. We would like to thank the UK Science and Technology Funding Council for supporting this work.

\section{References}

[1] Y. Itow et al., The JHF-Kamioka neutrino project, (2001), hep-ex/0106019

[2] Y. Fukuda et al., Nucl. Instrum. Meth. A501 418 (2003).

[3] Hamamatsu photonics, http://jp.hamamatsu.com

[4] J. Estrada, C. Garcia, B Hoeneison, P. Rubinov, MCM II and the Trip Chip, D0 Note 4009 (2002) 